\section{Commentary: Striving for higher level of evidence}

\author{
Shravan Chintalapani, MD, and Thomas Ng, MD
}

As thoracic surgery has become less and less invasive, attention has also turned to minimizing complications from conventional mechanical ventilation during surgery, including intubation injury, ventilation-induced injury, and sequela of muscle relaxants and opioid analgesia. Spontaneous ventilation video-assisted thoracic surgery (SVVATS) has emerged as a technique aimed to prevent these adverse effects of conventional mechanical ventilation video-assisted thoracic surgery. ${ }^{1}$ Liu and colleagues ${ }^{2}$ have performed a prospective randomized controlled trial to evaluate the perioperative safety and physiologic changes of these 2 ventilation strategies.

In this multicenter study, 335 patients with primary spontaneous pneumothorax were randomized to undergo uniportal bleb resection with SV-VATS or mechanical ventilation video-assisted thoracic surgery. Primary outcomes analyzed were intraoperative and postoperative complications including intraoperative hypoxia, conversion to intubation, air leak, and postoperative pneumonia. Secondary outcomes included total analgesic dose, changes in vital signs, blood gas analysis, operative time, recovery time, and length of hospitalization. Analysis showed no significant difference in primary outcomes between the 2 arms, with several of the secondary outcomes favoring SV-VATS.

Limitations of this study are few, but worth mentioning. First, there is potential bias through nonblinded anesthesia teams, noticeably regarding many of the secondary outcomes favoring SV-VATS, such as extubation time, postanesthesia care time, and intraoperative narcotic use. Second, the described protocol only offers regional anesthesia to the SV-VATS group, which possibly contributed

\footnotetext{
From the Division of Thoracic Surgery, University of Tennessee Health Science Center, College of Medicine, Memphis, Tenn.

Disclosures: The authors reported no conflicts of interest.

The Journal policy requires editors and reviewers to disclose conflicts of interest and to decline handling or reviewing manuscripts for which they may have a conflict of interest. The editors and reviewers of this article have no conflicts of interest.

Received for publication Feb 6, 2021; revisions received Feb 6, 2021; accepted for publication Feb 8, 2021; available ahead of print Feb 20, 2021.

Address for reprints: Thomas Ng, MD, Division of Thoracic Surgery, University of Tennessee Health Science Center, College of Medicine, 1325 Eastmoreland Ave, Suite 460, Memphis, TN 38104 (E-mail: tng4@uthsc.edu).

J Thorac Cardiovasc Surg 2022;163:1717-8

$0022-5223 / \$ 36.00$

Copyright (c) 2021 by The American Association for Thoracic Surgery

https://doi.org/10.1016/j.jtcvs.2021.02.047
}

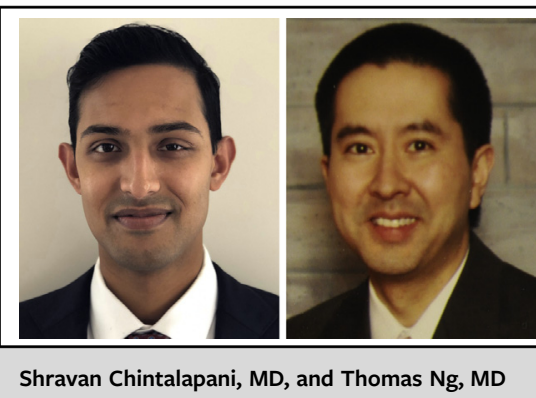

CENTRAL MESSAGE

Although no major difference was found, the authors are to be commended for using high-level evidence, conducting a randomized trial, to seek answers to their clinical question.

to the noticed decrease in intraoperative opiate use for this arm. Finally, without reporting long-term outcomes, we cannot determine if SV-VATS allows for an effective operation in terms of pneumothorax recurrence.

It is important to note that the population studied included only relatively young healthy patients, so generalization would be difficult. The safety of SV-VATS in older, less healthy patients is not known because the lower $\mathrm{pH}$, higher $\mathrm{pCO} 2$, higher heart rate, and lower blood pressure observed intraoperatively for the SV-VATS group may not be well tolerated in this patient population. Furthermore, the difficulty and potential danger of converting laryngeal mask to endotracheal tube in the lateral decubitus position, if SV-VATS fails, must be pointed out. One can argue these results only apply to simple thoracic procedures; however, Liu and colleagues ${ }^{2}$ note ongoing randomized trials examining SV-VATS for more complex surgeries.

Finally, although this randomized trial certainly has its limitations and did not show a difference in major outcomes, the authors should be commended for conducting a randomized study to try to answer a clinical question. Too often, we see lower-level evidence published that still leaves readers wondering if the question was answered. Much time and effort are needed to conduct randomized trials, but to be practice changing, there are rarely any other types of studies that can be a substitute for well-designed randomized trials. 


\section{References}

1. Wen Y, Liang H, Qiu G, Liu Z, Liu J, Ying W, et al. Non-intubated spontaneous ventilation in video-assisted thoracoscopic surgery: a meta-analysis. Eur J Cardiothorac Surg. 2020;57:428-37.

2. Liu J, Liang H, Cui F, Liu H, Zhu C, Liang W, et al. Spontaneous versus mechanical ventilation during video-assisted thoracoscopic surgery for spontaneous pneumothorax: a randomized trial. J Thorac Cardiovasc Surg. 2022;163:1702-14.e7. 